\title{
Measurement properties of the Minimal Insomnia Symptom Scale (MISS) in an elderly population in Sweden
}

\author{
Amanda Hellström ${ }^{1,2^{*}}$, Peter Hagell ${ }^{2}$, Cecilia Fagerström, Ania Willman ${ }^{1}$
}

\begin{abstract}
Background: Insomnia is common among elderly people and associated with poor health. The Minimal Insomnia Symptom Scale (MISS) is a three item screening instrument that has been found to be psychometrically sound and capable of identifying insomnia in the general population (20-64 years). However, its measurement properties have not been studied in an elderly population. Our aim was to test the measurement properties of the MISS among people aged $65+$ in Sweden, by replicating the original study in an elderly sample.

Methods: Data from a cross-sectional survey of 548 elderly individuals were analysed in terms of assumptions of summation of items, floor/ceiling effects, reliability and optimal cut-off score by means of ROC-curve analysis and compared with self-reported insomnia criteria.

Results: Corrected item-total correlations ranged between 0.64-0.70, floor/ceiling effects were $6.6 / 0.6 \%$ and reliability was 0.81 . ROC analysis identified the optimal cut-off score as $\geq 7$ (sensitivity, 0.93 ; specificity, 0.84; positive/ negative predictive values, $0.256 / 0.995)$. Using this cut-off score, the prevalence of insomnia in the study sample was $21.7 \%$ and most frequent among women and the oldest old.

Conclusions: Data support the measurement properties of the MISS as a possible insomnia screening instrument for elderly persons. This study make evident that the MISS is useful for identifying elderly people with insomnia-like sleep problems. Further studies are needed to assess its usefulness in identifying clinically defined insomnia.
\end{abstract}

\section{Background}

According to the International Classification of Sleep Disorders, second edition (ISCD-2), the diagnosis of insomnia is based on a subjective report of difficulties initiating sleep, maintaining sleep due to night time or early morning waking or non-restorative sleep, despite ideal sleep conditions, leading to daytime impairments [1-3]. Secondary insomnia is the most common form and can be related to an underlying somatic or psychic disease or pharmaceutical use [4].

Many elderly people suffer from one or more chronic medical conditions that can cause or contribute to sleep difficulties [5]. Persistent insomnia in elderly persons is often associated with co-morbidity, use of medication or other primary sleep disorders [6]. Common sleep

\footnotetext{
* Correspondence: amanda.hellstrom@bth.se

'School of Health Science, Blekinge Institute of Technology, SE-371 79 Karlskrona, Sweden

Full list of author information is available at the end of the article
}

difficulties among elderly people include waking at night and longer periods of wakefulness before falling asleep again [7-9].

Sleep difficulties, such as persistent insomnia, are associated with immune system changes [10], the development of diseases such as depression as well as impaired memory function and ability to concentrate and make decisions $[11,12]$. Consequently insomnia also has societal and economic implications and is for example associated with an elevated risk of disability pension [13]. However, while a significant minority of the adult population has at least one insomnia symptom occurring three nights or more per week, the majority do not seek help. This can be explained by lack of knowledge about insomnia in the general population but also by under-recognition and under-diagnosis on the part of health care personnel. It is important to take symptoms of insomnia seriously [14], since they tend to become persistent [1]. 
In general, insomnia has been found to increase with age and is more common among women [7]. In persons aged 65 years or over, the risk of developing insomnia is $73 \%$ greater in women than men [15]. Elderly people are a vulnerable group, and long questionnaires may be perceived as tiresome to fill in, not only by the elderly individuals themselves but also by health care personnel. A short screening instrument that could identify persons with self-reported insomnia would therefore be useful.

The Minimal Insomnia Symptom Scale (MISS) was developed from a more extensive sleep questionnaire and is a brief, three item screening instrument [1]. The basic psychometric properties of the MISS were evaluated in a randomly selected general population ( $\mathrm{n}=$ 1075) aged between 20 and 64 years, and the prevalence of insomnia was found to be $22.5 \%$. Although the findings support the measurement properties of the MISS as an insomnia screening instrument, the extent to which these data are applicable to an elderly population is still unknown. Our aim was to test the measurement properties of the MISS among people aged $65+$ in Sweden, by replicating the original study [1] in an elderly sample.

\section{Methods}

\section{Design and study sample}

The study, which had a cross-sectional explorative design, was conducted within the Swedish National Study on Ageing and Care - Blekinge (SNAC-B) longitudinal study, which is part of the Swedish National Study on Ageing and Care [16]. The SNAC-B population consists of elderly persons living in Karlskrona municipality, which is situated in the south-east of Sweden and comprises both urban and rural areas. When the SNAC-B was launched in 2001, 1402 persons agreed to participate. The SNAC-B target sample, $59.4 \%$ of which were women, included ten age clusters representing the ageing population of Sweden. There was a randomized selection of the younger age clusters $(60,66,72$ and 78 year olds) as well as a total inclusion of the clusters of people aged 81, 84, 87, 90, 93 and 96 years at baseline [16]. In 2008, when the data collection for the present study took place, 978 persons were still alive and registered as participants, of whom 892 were available for participation.

The original ten age clusters used in SNAC-B were amalgamated into three clusters for this study; 65-74 years (including the 67 and 73 year olds), 75-89 years (including the 79, 85 and 88 year olds) and 90+ (including those aged 91 years or older). The distribution of individuals across the age clusters and in terms of gender is described in Table 1 . Approximately $19.1 \%$ of the population of Karlskrona municipality is 65 years or older, which is a slightly higher age composition compared to Sweden as a whole [16].
Table 1 Distribution of the study sample

\begin{tabular}{llll}
\hline Age-group & Male (\%) & Female (\%) & Total (\%) \\
\hline $65-74$ & $119(51.1)$ & $146(46.3)$ & $265(48.4)$ \\
$75-89$ & $92(39.5)$ & $131(41.6)$ & $223(40.7)$ \\
$90+$ & $22(9.4)$ & $38(12.1)$ & $60(10.9)$ \\
\hline Total & $\mathbf{2 3 3 ( 4 2 . 5 )}$ & $\mathbf{3 1 5}(\mathbf{5 7 . 5 )}$ & $\mathbf{5 4 8 ( 1 0 0 )}$ \\
\hline
\end{tabular}

\section{Procedure}

The study was approved by the regional Ethical Review Board in Lund (LU 605-00, LU 744-00, 178/2008). In December 2008, questionnaires were mailed to the 892 persons participating in the SNAC-B study together with a consent form and a pre-stamped response envelope. Six weeks later, a reminder was sent to those who had not yet returned the questionnaire. All participants had the possibility to withdraw from the study at any time without having to give a reason and were assured of confidentiality.

\section{Questionnaire}

The survey consisted of the Uppsala Sleep Inventory 25 (USI-25) and three additional questions concerning age, sex and ability to fill out the form (independently, with help from a relative/friend or home care personnel).

The USI-25 is a 25 item self-report questionnaire about sleep difficulties and habits [1] previously used in studies of sleep associated with specific health conditions [17-19], among the elderly [20] and in longitudinal studies $[21,22]$. The USI-25 includes MISS, which consists of three items describing the major features of insomnia, i.e. difficulties initiating sleep, waking at night and not feeling refreshed by sleep [1]. Each item has five response alternatives; no problem (0), small problems (1), moderate problems (2), severe problems (3) and very severe problems (4). This yields a total score ranging between 0 and 12, where higher scores indicate more severe insomnia. A cut-off score of $\geq 6$ on the MISS has previously been suggested for identifying insomniacs in the general adult population between 20 and 64 years old (sensitivity 0.82 ; specificity 0.86 ; positive predictive value 0.44 ; negative predictive value 0.95 ). The average item-total correlation was 0.55 and reliability (coefficient alpha) was 0.73 . Validation against selfreported indices of insomnia criteria (confirmatory answers about suffering from sleep difficulties at least three times per week for a minimum duration of one month in addition to major daytime impairments due to poor sleep) supported the instrument's validity [1].

\section{Data analysis}

Analyses were conducted using SPSS 17.0, (SPSS Inc Chicago, IL.) P-values $<0.05$ were considered statistically significant. 
First, assumptions concerning adding up item scores into a total score in accordance with the Likert tradition were tested, i.e. item mean scores, standard deviations and corrected item-total correlations should be fairly similar across items. Furthermore, there should be evident that items represent a common variable. This was considered supported if corrected item-total correlations were $\geq 0.4$ [23]. Floor and ceiling effects (i.e., the proportion of people with minimum and maximum scores respectively) and score reliability (coefficient $\alpha$ ) were then calculated. Floor and ceiling effects should not exceed 15\% [24] and reliability should be between 0.7 and 0.9 [25].

We then examined the ability of the MISS scores to identify persons with self-reported insomnia and explored different cut-off scores. A criterion variable was constructed by amalgamating three USI-items regarding daytime sleepiness, feeling physically tired after sleep and experiencing sleep difficulties. This variable served as a proxy gold standard against which the MISS scores could be compared. Respondents who reported the presence of severe or very severe problems on these items were considered to suffer from insomnia. Although our criterion variable differs from that used in the original MISS publication [1], it agrees with some of the insomnia criteria described in the ICSD-2, such as daytime sleepiness, fatigue and concerns about sleep [3]. A receiver operating characteristic (ROC) curve was then drawn and the area under the ROC curve (AUROC) was calculated. The ROCcurve illustrates the discriminating abilities of a test at different cut-off values [26]. Based on the ROC-curve, sensitivity and specificity were calculated for various cut-off scores. Sensitivity is the ability to diagnose a condition correctly, while specificity measures the ability to accurately identify non-cases. These values provide an indication of the discriminatory abilities of the scale [27]. In addition, the positive predictive value (PPV) and negative predictive value (NPV) were calculated. These values depend not only on sensitivity and specificity but also on the prevalence of the condition in question $[27,28]$. PPV and NPV represent the probability of a certain outcome when the screening results (i.e. whether scores are above or below a certain cutoff) are known.

When sensitivity and specificity are considered equally important, Youden's index (sensitivity + specificity -1) can be used to choose an appropriate cut-off value. Since the maximum value of Youden's index is 1 , indicating a perfect test, the cut-off score associated with the highest Youden index $(\mathrm{J})$ is considered optimal [29]. Finally, the prevalence of insomnia (including 95\% confidence intervals) according to the MISS was determined.

\section{Results}

\section{Description of the study sample}

Of the 892 questionnaires, 548 were returned together with a signed informed consent form, yielding a response rate of $61.4 \%$ (Figure 1 ). The mean age of the respondents was 77.8 (SD 8.63) years. The distribution of respondents across genders and the three age clusters are presented in Table 1. Common reasons for declining participation were advanced age, illness, vision disability, being unable to obtain help to fill in the questionnaire or feeling tired. The external drop out (Table 2) mainly represents persons of advanced age and those with cardiovascular disease or type- 2 diabetes. Most of the participants $(n=479 ; 87.5 \%)$ completed the questionnaire on their own, while 66 (12\%) obtained help from a relative or friend and three $(0.5 \%)$ received assistance from health care personnel.

Responses to the USI-25 items revealed that close to a third $(28.5 \%)$ of the sample considered themselves to suffer from sleep difficulties ( 9 persons failed to answer this question). Regular use of sleep medication was found to increase with age; $5.0 \%$ of those aged $65-74$ years used sleep medication very often, while the corresponding figure among the oldest old was $24.1 \%$. There

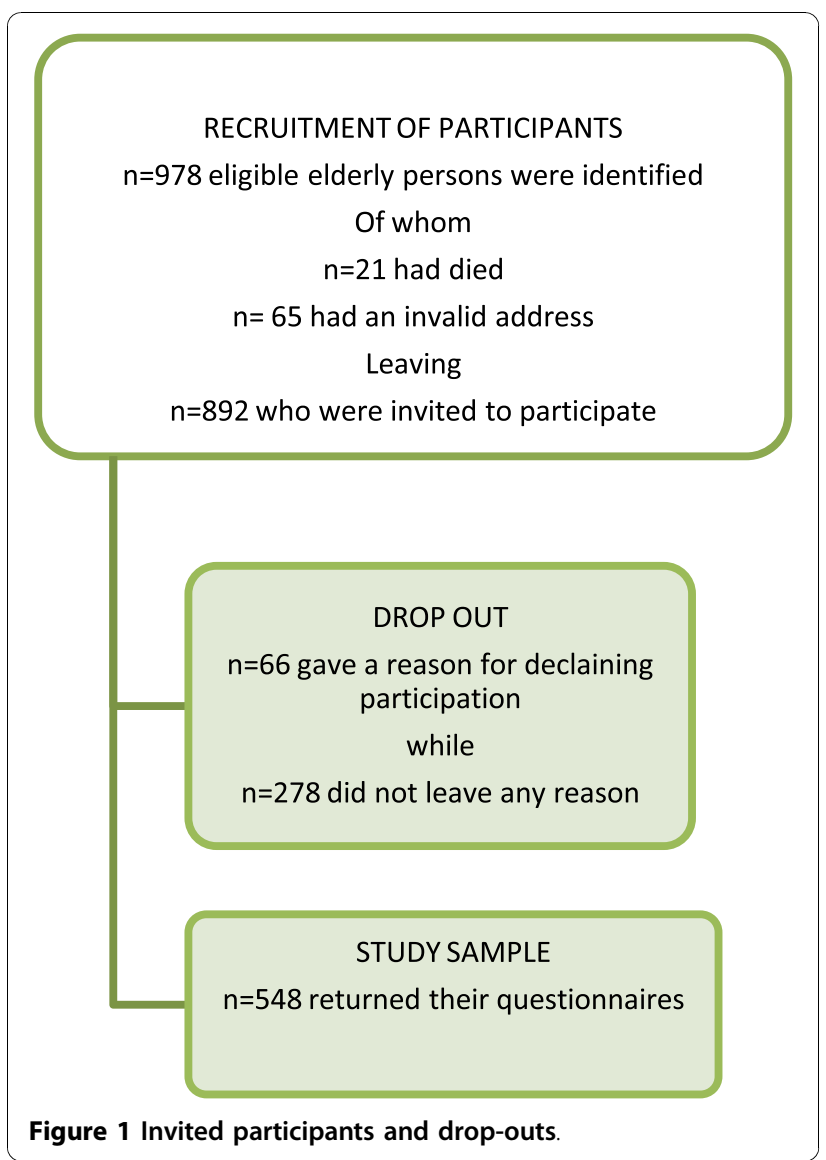


Table 2 Characteristics of non-participants vs. participants

\begin{tabular}{llll}
\hline Variables & $\begin{array}{l}\text { Non-participants } \\
(\mathbf{n = 3 4 4 )}\end{array}$ & $\begin{array}{l}\text { Participants } \\
(\mathbf{n}=\mathbf{5 4 8})\end{array}$ & p-value $^{\mathbf{1}}$ \\
\hline Age mean (SD) & $83.2(9.8)$ & $77.8(8.6)$ & $<0.001$ \\
Sex $\quad$ Female \% & 39.9 & 60.1 & 0.302 \\
$\quad$ Male \% & 36.2 & 63,8 & \\
& & & \\
Angina Pectoris \% & 48.8 & 51.2 & 0.014 \\
& & & \\
Arthritis \% & 43.0 & 57.0 & 0.183 \\
Cancer \% & 43.0 & 57.0 & 0.328 \\
Chronic heart failure \% & 62.2 & 37.8 & 0.001 \\
& & & \\
Depression \% & 43.9 & 56.1 & 0.236 \\
Diabetes type 2 \% & 53.8 & 46.2 & 0.012 \\
Hypertension \% & 44.8 & 55.2 & 0.019 \\
& & & \\
Osteoporosis \% & 55.2 & 44.8 & 0.084 \\
\hline
\end{tabular}

1) Chi-square test and Mann-Whitney U-test. For the Chi-square test Yates' correction has been used.

Internal drop-out on variables was $0-1.2 \%$ accept for osteoporosis (10.5\%) and arthritis (17.1\%).

was also a predominance of women among sleep medication users. The highest proportion of daytime impairments (i.e., daytime sleepiness, feeling physically tired after sleep and reporting sleep difficulties) was found among the $65-74$ year olds (7.1\%), whereas only $3.8 \%$ of the oldest old reported such impairments. Interestingly, of those who claimed to have no sleep difficulties, 317 reported insomnia symptoms on the MISS. There were more women than men that reported insomnia symptoms (Table 3).

Measurement properties of the MISS

Fifty-one persons (9.3\%) failed to fill in all the MISS items, thus complete MISS data were obtained from a total of 497 individuals. Mean MISS item scores ranged

Table 3 Sleep difficulties found among the elderly persons

\begin{tabular}{llllll}
\hline Sleep difficulty & Female & Male & $\mathbf{6 5 - 7 4}$ & $\mathbf{7 5 - 8 9}$ & $\mathbf{9 0 +}$ \\
\hline Difficulties initiating sleep \% & 18.6 & 9.1 & 13.8 & 15.5 & 13.8 \\
Difficulties maintaining sleep \% & 22.3 & 15.6 & 19.5 & 18.7 & 21.4 \\
Not being refreshed \% & 18.2 & 12.8 & 17.8 & 14.2 & 13.2 \\
No insomnia symptoms \% & 5.4 & 8.3 & 5.8 & 8.9 & 1.9 \\
One insomnia symptom \% & 16.8 & 12.0 & 13.4 & 16.4 & 13.2 \\
Two insomnia symptoms \% & 10.4 & 6.9 & 9.1 & 8.9 & 7.5 \\
Three insomnia symptoms \% & 6.4 & 3.7 & 6.2 & 3.9 & 5.7 \\
\hline
\end{tabular}

Insomnia symptom = reports of severe/very severe problems with initiating sleep, maintaining sleep or not being refreshed by sleep. between 1.2-1.7 (SD 1.0-1.2) and corrected item-total correlations between 0.64-0.70. Inter-item correlation ranged between $0.55-0.62$. Reliability was 0.81 . Floor and ceiling effects were 6.6 and $0.6 \%$, respectively.

The criterion validity of the MISS was tested against the amalgamated criterion variable which corresponds with some of the diagnostic criteria in the ICSD-2 [3]. The MISS ROC-curve is presented in Figure 2. The area under the curve was 0.935 (95\% CI: 0.90-0.97; P < 0.001 ), indicating good correspondence between the criterion variable and the MISS. According to the Youden index, a cut-off MISS score of $\geq 7$ was found the most optimal (Table 4).

\section{Prevalence of insomnia}

The mean MISS score for the total sample was 4.11 (95\% CI, 3.86-4.35). The prevalence of self-reported insomnia in this sample of elderly persons based on various MISS cut-off scores can be seen in Table 5. Using a cut-off of $\geq 7$, the overall prevalence of insomnia was $21.7 \%$ (95\% CI, 18-25\%). Insomnia tended to be more common among persons aged 90 years or over (24.1\%) and affected more women (26.8\%) than men (15.2\%). Among those identified as insomniacs, $12.3 \%$ answered "no" to the question of perceived sleep difficulties.

\section{Discussion}

This study tested the measurement properties of a brief insomnia screening instrument, the MISS, among elderly persons. Previous testing of the MISS in the general population under 65 years of age, found it to be sound

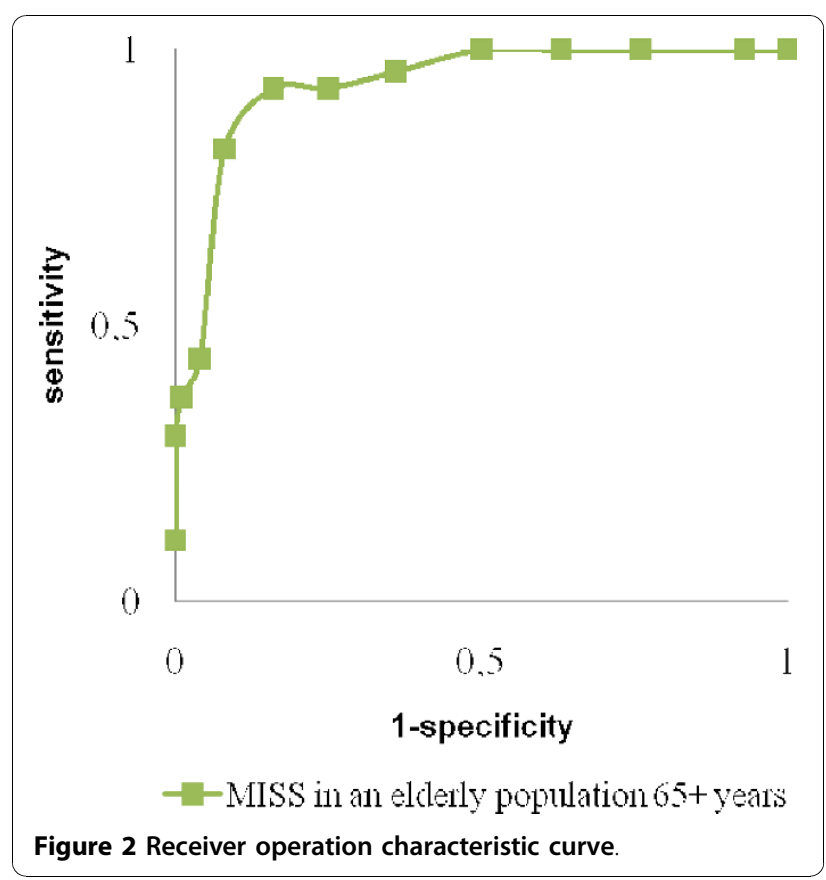


Table 4 MISS cut-off values, sensitivity, specificity, positive predictive value (PPV), negative predictive value (NPV) and Youden's index

\begin{tabular}{lllllll}
\hline Cut-off score & Sensitivity & Specificity & 1-specificity & PPV & NPV & Youden's index \\
\hline$\geq 5$ & 0.963 & 0.643 & 0.357 & 0.137 & 0.997 & 0.606 \\
$\geq 6$ & 0.926 & 0.752 & 0.248 & 0.181 & 0.994 & 0.678 \\
$\geq 7$ & 0.926 & 0.838 & 0.162 & 0.256 & 0.995 & 0.764 \\
$\geq 8$ & 0.815 & 0.920 & 0.080 & 0.377 & 0.988 & 0.730 \\
\hline
\end{tabular}

and capable of identifying insomnia. This study provides further support for the usefulness of the MISS and extends its application to include elderly persons. However, our findings differ somewhat from the previous study in terms of suggested cut-off score and diagnostic ability. Based on available MISS data we also consider possible developments of the MISS in order to enhance its usefulness, by specifying the response alternatives and add a fourth item comprising daytime impairments.

The screening capacity of the MISS in this study (AUROC, 0.94; 95\%CI, 0.9-0.97) was similar to that reported in a younger adult population (AUROC, 0.92; 95\%CI, 0.89-0.94) [1]. But a cut-off value $\geq 7$ was found more suitable than that of $\geq 6$ previously identified in younger adults [1]. This divergence may be due to the variation in size and age of the study samples, as well as the use of different gold standards. It is possible that healthy, elderly persons perceive disturbed sleep and daytime impairments as related to the advanced age. The proxy-gold standard used in this study prerequisite recognition of daytime impairments. There is thus a risk that the prevalence of insomnia is under-recognized. This would also have bearing on the reported positive predicted value. Similarly to the previous study [1] MISS scores were not directly tested against diagnostic insomnia criteria but a proxy gold standard was used instead. However, the proxy gold standard was not the same as in the original study. While this may explain the observed differences in result, future studies should consider testing the MISS directly against diagnostic insomnia criteria in order to provide support for the legitimacy of creating a total MISS score that is reliable, unidimensional and valid in people aged 65 years or older.

A cut-off value of $\geq 7$ revealed a $21.7 \%$ prevalence of insomnia in the present study. In previous testing of the
MISS in the general population using a cut-off value of $\geq 6$, the prevalence of insomnia was found to be $22.5 \%$ [1]. This shows a similarity between the studies in estimating the prevalence of insomnia symptoms. Other studies have found that insomnia symptoms occur in $33-50 \%$ of the adult population, while the prevalence of insomnia disorder has been estimated to about $10-30 \%$ [14,30-32]. In relation to these figures, the MISS appears to blunt for diagnosing insomnia.

Therefore, if the goal is to diagnose insomnia, additional investigations based on clinical diagnostic criteria are needed, as pointed out by the constructors of the MISS [1]. The MISS should thus be regarded as an initial screening instrument. Although its brevity is advantageous in the inclusion of only three items also limits the usefulness of the MISS. It appears reasonable to suggest that the MISS would benefit from adding a fourth item concerning daytime impairments which is among the diagnostic criteria for insomnia [3]. Similarly, it could also be considered to incorporate some references to the duration and frequency of the sleep difficulties specified by the MISS items, possibly by integrating this with the response options. Compared to other available short insomnia screening questionnaires, which contain 6-10 items [33-35], such an expansion of the MISS would still render the briefest insomnia screening questionnaire.

Healthy elderly persons adjust their expectations of sleep, accepting the changes as a normal part of aging. It has been suggested that it is the individual's perspective of growing old that is significant for the experience of good or poor sleep. The presence of a medical disease or chronic illness has stronger correlations with poor sleep than chronological age per se [36,37]. Women also appear to accept sleep loss as a natural part of life. Sociologists consider that women indeed recognize sleep

Table 5 Prevalence of insomnia according to different MISS cut-off scores

\begin{tabular}{cccccccc}
\hline Cut-off & $\begin{array}{c}\text { Mean score } \\
(\mathbf{9 5 \% C l )}\end{array}$ & $\begin{array}{c}\text { All } \\
(\mathbf{n = 4 9 7 )}\end{array}$ & $\begin{array}{c}\text { Male } \\
(\mathbf{n = 2 1 7 )}\end{array}$ & $\begin{array}{c}\text { Female } \\
(\mathbf{n}=\mathbf{2 8 0})\end{array}$ & $\begin{array}{c}\mathbf{6 5 +} \\
(\mathbf{n}=\mathbf{2 4 2})\end{array}$ & $\begin{array}{c}\mathbf{7 5 +} \\
(\mathbf{n}=\mathbf{2 0 1})\end{array}$ & $\begin{array}{c}\mathbf{9 0 +} \\
(\mathbf{n}=\mathbf{5 4})\end{array}$ \\
\hline$\geq 5$ & $6.91(6.67-7.15)$ & $202(40.6 \%)$ & $69(31.8 \%)$ & $133(47.5 \%)$ & $99(40.9 \%)$ & $82(40.8 \%)$ & $21(38.9 \%)$ \\
$\geq 6$ & $7.57(7.33-7.81)$ & $150(30.2 \%)$ & $51(23.5 \%)$ & $99(35.4 \%)$ & $71(29.3 \%)$ & $62(30.8 \%)$ & $17(31.5 \%)$ \\
$\geq 7$ & $8.19(7.94-8.44)$ & $108(21.7 \%)$ & $33(15.2 \%)$ & $75(26.8 \%)$ & $52(21.5 \%)$ & $43(21.4 \%)$ & $13(24.1 \%)$ \\
$\geq 8$ & $8.97(8.68-9.26)$ & $65(13.0 \%)$ & $21(9.6 \%)$ & $44(15.7 \%)$ & $32(13.2 \%)$ & $23(11.4 \%)$ & $10(18.5 \%)$ \\
\hline
\end{tabular}


as essential for good health, but the demands of their social roles determines to which extent they can access adequate sleep. Over time the social roles in life of the women, conceal the sleep disruption until poor sleep patterns becomes the norm [38]. This could imply that women and elderly persons are inattentive to insomnia symptoms, contributing to the under-diagnosis of the disorder. Our findings reveal that insomnia is more prevalent in women and in the oldest old. However, the MISS is a self-report questionnaire, reflecting subjective experiences rather than the prevalence of insomnia disorder, which is problematic when comparing groups. Objective measures could yield a different result [39]. Furthermore, previous research has found that selfreports of polysomnographically measured sleep are less accurate and valid in older persons compared to younger [36].

\section{Limitations}

There are limitations in the design of the study that need to be considered. A concern arose when using the USI-25 questionnaire, particularly when trying to validate the MISS, as the former contains no measure of the duration or frequency of sleep difficulties, nor daytime impairments. This makes it difficult to establish a clinical diagnosis of insomnia, as such a diagnosis is based on the duration of the sleep difficulty, impaired daytime functioning and the three major symptoms [3]. However, it was considered important not to increase respondent burden by using multiple questionnaires, due to the vulnerability of the study sample and the risk of further compromising the response rate.

Another limitation was the cross-sectional design. Repeated measures could have contributed to more robust reliability and validity [40]. Furthermore, the study design did not allow for any testing of sensitivity to change in order to verify or reject previous findings [1], which have provided some support for responsiveness of the MISS.

As the respondents participated in the SNAC-B study, we had access to data about the prevalence of different diseases in the sample, which was used when analysing the external drop out. The external drop-out analysis revealed that persons with cardiovascular disease and diabetes, who could be expected to suffer from insomnia $[41,42]$, chose to decline participation, which may have influenced the findings. Furthermore, the MISS has not been tested as an independent instrument but was extracted from the USI-25 questionnaire, which may have implications for the response rate in the present study. Finally, future studies on the MISS should consider using more advanced psychometric methods, such as Rasch analysis or item response theory.

\section{Conclusion}

This is the first study of the measurement properties of the MISS in an elderly population, and our observations provide support for its reliability and validity in elderly persons in Sweden. As such, the results provides further support that the MISS is useful for identifying elderly persons with insomnia-like sleep problems who would be candidates for more detailed assessments. However, further studies are needed to assess its usefulness in identifying clinically defined insomnia.

\section{Acknowledgements}

We express our gratitude to the study participants. The Swedish National study on Aging and Care (SNAC) is financially supported by the Ministry of Health and Social Affairs, Sweden, and by the participating county councils, municipalities and university departments. We also thank the School of Health Science, Blekinge Institute of Technology, for supporting the study.

\section{Author details}

'School of Health Science, Blekinge Institute of Technology, SE-371 79 Karlskrona, Sweden. ${ }^{2}$ Department of Health Sciences, Lund University, Lund, Sweden.

\section{Authors' contributions}

AH was responsible for data collection, data analysis and drafting of the manuscript. PH contributed to the design and conception of the study, statistical expertise, critical revision and important intellectual content. CF helped with the statistics, the design and conception of the study and also performed critical revisions and supervision. AW was involved in the drafting of the manuscript, critical revisions, important intellectual content and supervision. All of the authors have read and approved the final manuscript.

\section{Competing interests}

The authors declare that they have no competing interests.

Received: 11 June 2010 Accepted: 5 November 2010

Published: 5 November 2010

\section{References}

1. Broman J-E, Smedje H, Mallon L, Hetta J: The minimal insomnia symptom scale (MISS): a brief measure of sleeping difficulties. Uppsala J Med Sci 2008, 113(2):131-142.

2. Ohayon MM: Epidemiology of insomnia: what we know and what we still need to learn. Sleep Med Rev 2002, 6(2):97-111.

3. Schutte-Rodin S, Broch L, Buysse D, Dorsey C, Sateia M: Clinical guideline for the evaluation and management of chronic insomnia in adults. J Clin Sleep Med 2008, 4(5):487-504.

4. Wolkove N, Elkholy O, Baltzan M, Palayew M: Sleep and ageing:1. Sleep disorders commonly found in older people. Can Med Assoc J 2007, 176(9):1299-1304.

5. Ancoli-srael S, Cooke JR: Prevalence and comorbidity of insomnia and effect on functioning in elderly populations. J Am Geriatr Soc 2005, 53(7):264-271.

6. Stone KL, Ensrud KE, Ancoli-Israel S: Sleep, insomnia and falls in elderly patients. Sleep Med 2008, 9(1):18-22.

7. Foley DJ, Monjan AA, Brown SL, Simonsick EM, Wallace RB, Blazer DG: Sleep complaints among elderly persons: an epidemiologic study of three communities. Sleep 1995, 18(6):425-432.

8. Roehrs T: Sleep physiology and pathophysiology. Clin Cornerstone 2000, 2(5):1-12.

9. Åkerstedt T, Billiard M, Bonnet M, Ficca G, Garma L, Marilotti M, Salzarulo P, Schultz H: Awakening from sleep. Sleep Med Rev 2002, 6(4):267-286.

10. Savard J, Laroche L, Simard S, Ivers H, Morin CM: Chronic insomnia and immune functioning. Psychosom Med 2003, 65:211-221.

11. Broman J-E: Den normala sömnen [The normal sleep]. In Sömn 2009 Edited by: Ulfberg J. Falun: Circad bok; 2009:15-22. 
12. Hoffman S: Sleep in the older adult: Implications for nurses. Geriatr Nurs 2003, 24(4):210-216.

13. Sivertsen B, Overland S, Pallesen S, Bjornvatn B, Nordhus IH, Maeland JG, Mykletun $A$ : Insomnia and long sleep duration are risk factors for later work disability. The Hordaland Health Study. I Sleep Res 2009, 18:122-128.

14. Morin CM, LeBlanc M, Daley M, Gregoire JP, Mérette C: Epidemiology of insomnia: prevalence, self-help treatments, consultations, and determinants of help-seeking behaviors. Sleep Med 2006, 7:123-130.

15. Philips BA, Collop NA, Drake C, Consens F, Vgontzas AN, Weaver TE: Sleep disorders and medical conditions in women. Proceedings of the women and sleep workshop, National Sleep Foundation, Washington, DC, March 5-6 2007. J Women's Health 2008, 17(7):1191-1199.

16. Lagergren M, Fratiglioni L, Hallberg IR, Berglund J, Elmståhl S, Hagberg B, Holst G, Rennemark M, Sjölund B-M, Thorslund M, et al: A longitudinal study integrating population, care and social service data. The Swedish National study on Aging and Care (SNAC). Aging Clin Exp Res 2004, 16(2):158-168.

17. Edéll-Gustafsson UM, Hetta JE: Anxiety, depression and sleep in male patients undergoing coronary artery bypass surgery. Scand I Caring Sci 1999, 13:137-143.

18. Edéll-Gustafsson UM, Gustavsson G, Yngman-Uhlin P: Effects of sleep loss in men and women with insufficient sleep suffering from chronic disease: a model for supportive nursing care. Int I Nurs Pract 2003, 9: 49-59.

19. Yngman-Uhlin P, Edéll-Gustafsson U: Self-reported subjective sleep quality and fatigue in patients with perioneal dialysis treatment at home. Int $J$ Nurs Pract 2006, 12: 143-152.

20. Mallon L, Hetta J: A survey of sleep habits and sleeping difficulties in an elderly Swedish population. Uppsala J Med Sci 1997, 102:185-197.

21. Mallon L, Broman J-E, Hetta J: Relationship between insomnia, depression and mortality: a 12-year follow-up of older adults in the community. Int Psychogeriatr 2000, 12(3):295-306.

22. Mallon L, Broman J-E, Hetta J: Sleep complaints predict coronary artery disease mortality in males: a 12-year follow-up study of a middle-aged Swedish population. J Intern Med 2002, 251: 207-216.

23. Ware JE, Gandek B: Methods for testing data quality, scaling assumptions and reliability: The IQOLA Project Approach. J Clin Epidemiol 1998, 51(11):945-952.

24. McHorney CA, Tarlov AR: Individual-patient monitoring in clinical practice: are available health status surveys adequate? Qual Life Res 1995, 4:293-307.

25. Aday LU, Cornelius LJ: Designing and conducting health surveys. A comprehensive guide. San Fransisco: Jossey-Bass; 32006.

26. Goutham R: What is an ROC curve. J Fam Pract 2003, 52(9):695.

27. Bring J, Taube A: Introduktion till medicinsk statistik [Introduction to medical statistics]. Lund: Studentlitteratur; 2006.

28. Polit $D E$, Beck $C T$ : Nursing research. Generating and assesing evidence for nursing practice. Philadelphia: Lippincott Williams \& Wilkins; 82008.

29. Bewick V, Cheek L, Ball J: Statistics review 13: Receiver operating characteristic curves. Crit Care 2004, 8(6):508-512.

30. Ohayon MM, Paiva T: Global sleep dissatisfaction for the assessment of insomnia severity in the general population of Portugal. Sleep Med 2005, 6:435-441.

31. Pallesen $\mathrm{S}$, Nordhus $\mathrm{IH}$, Nielsen $\mathrm{GH}$, Havik OE, Kvale G, Johnsen BH, Skjötskift S: Prevalence of insomnia in the adult Norweigan population. Sleep 2001, 24(7):771-779.

32. Redondo-Martínez MP, Salcedo-Aguilar F, García-Jiménez MA, MonterdeAznar ML, Rodríguez-Almonacid FM, Marcos-Navarro Al: Prevalencia de insomnio y consumo de psicofármacos en ancianos de una zona básica de salud de Cuenca. [Prevalence of insomnia and use of psychodrugs among elderly in a basic health area in Cuenca]. Aten Primaria 2000, 25(6):400-404.

33. Bastien $\mathrm{CH}$, Vallières $\mathrm{A}$, Morin $\mathrm{CM}$ : Validiation of the Insomnia Severity Index as an outcome measure for insomnia research. Sleep Med 2001, 2:297-307.

34. Pallesen S, Bjorvatn B, Nordhus $I H$, Sivertsen B, Hjornevik M, Morin CM: A new scale for measuring insomnia: the Bergen Insomnia Scale. Percept Mot Skills 2008, 107(3):691-706.

35. Soldatos CR, Dikeos DG, Paparrigopoulos TJ: Athens Insomnia Scale: validation of an instrument based on ICD-10 criteria. J Psychosom Res 2000, 48:555-560.
36. Bliwise DL: Sleep in normal ageing and dementia. Sleep 1993, 16(1):40-81.

37. Zilli I, Ficca G, Salzarulo P: Factors involved in sleep satisfaction in the eldely. Sleep Med 2009, 10:233-239.

38. Dzaja A, Arber S, Hislop J, Kerkhofs M, Kopp C, Pollmächer T, Polo-Kantola P, Skene D, Stenuit P, Tobler I, et al: Women's sleep in health and disease. J Psychiatr Res 2005, 39:55-76.

39. Berg JFvd, Rooij Fv, Vos H, Tulen J, Hofman A, Miedema H, Neven AK, Tiemeier $\mathrm{H}$ : Disagreement between subjective and actigraphic measures of sleep duration in a population-based study of elderly persons. J Sleep Res 2008, 17:295-302.

40. Streiner DL, Norman GR: Health measurement scales. A practical guide to their development and use. Oxford: Oxford University Press; 42008.

41. Ancoli-lsrael S: The impact and prevalence of chronic insomnia and other sleep disturbances associated with chronic illness. Am J Manag Care 2006, 12(8):S221-S229.

42. Edéll-Gustafsson U, Svanborg E, Swahn E: A gender perspective on sleeplessness behavior effects of sleep loss, and coping resources in patients with stable coronary artery disease. Heart lung 2006, 35(2):75-89.

\section{Pre-publication history}

The pre-publication history for this paper can be accessed here: http://www.biomedcentral.com/1471-2318/10/84/prepub

doi:10.1186/1471-2318-10-84

Cite this article as: Hellström et al:: Measurement properties of the Minimal Insomnia Symptom Scale (MISS) in an elderly population in Sweden. BMC Geriatrics 2010 10:84.

\section{Submit your next manuscript to BioMed Central and take full advantage of:}

- Convenient online submission

- Thorough peer review

- No space constraints or color figure charges

- Immediate publication on acceptance

- Inclusion in PubMed, CAS, Scopus and Google Scholar

- Research which is freely available for redistribution 